\title{
Locating Conical Intersections Using the Quasidegenerate Partially and Strongly Contracted NEVPT2 Methods
}

\author{
Yoshio Nishimoto $^{\mathrm{a}}$ \\ ${ }^{a}$ Institute for Fundamental Chemistry, Kyoto University, 34-4 Takano Nishihiraki-cho, \\ Sakyo-ku, Kyoto 606-8103, Japan
}

\begin{abstract}
Conical intersections (CIs) play an important role in photochemistry because they sometimes govern the non-radiative decay process. However, accurate characterizations of CIs are not always straightforward. In this study, analytic gradients and interstate coupling vectors for the quasidegenerate partially and strongly contracted $n$-electron valence state second-order perturbation theory (QD-PC-NEVPT2 and QD-SC-NEVPT2) were developed and applied to locating CIs of benzene. The pilot application demonstrates that the results of both the methods are similar to the extended multistate complete active space second-order perturbation theory (XMS-CASPT2), while the lack of dynamic electron correlation resulted in a deviation of 0.7 $\mathrm{eV}$.

Keywords: multireference perturbation theory, $n$-electron valence state second-order perturbation theory (NEVPT2), conical intersections, analytic derivatives
\end{abstract}

\section{Introduction}

Crossings between potential energy surfaces of different states play important roles in photochemistry $[1,2,3]$. Those between same spin states and spatial symmetries are usually referred to as conical intersections (CIs)

\footnotetext{
${ }^{*}$ Corresponding author. Fax: $\quad+81 \quad(0) 75 \quad 711-7894$, e. $\quad$ mail nishimoto.yoshio@fukui.kyoto-u.ac.jp 
and are responsible for non-radiative transitions between states. In particular, minimum energy CIs (MECIs) serve as an efficient "funnel" around the CI region, and the higher state efficiently and non-radiatively decays to the lower state. Many studies have used the mean-field approach, i.e., multiconfiguration self-consistent field (MCSCF) and its complete active space variant (CASSCF) [4]; however, the lack of dynamic electron correlation is a major issue.

Dynamic electron correlation can be adequately described with the multireference coupled-cluster (MRCC) [5, 6, 7], configuration interaction (MRCI) [8], or perturbation theories (MRPTs). MRCC and MRCI are usually the preferred methodologies used to describe ground and excited states very accurately; however, the drawback of these methods is the expensive computational cost. Considering the required balance between computational cost and accuracy, MRPT methods are possible solutions. Recent studies [9] on complete active space second-order perturbation theory (CASPT2) $[10,11,12]$ has made it possible to explore CI regions using analytic derivative coupling vectors [13] at the extended multistate (XMS) extension $[14,15]$ of CASPT2 (XMS-CASPT2). Non-adiabatic molecular dynamics simulations can also be performed at the XMS-CASPT2 level of theory [16]. The second-order generalized Van Vleck perturbation theory (GVVPT2) [17] was also used to exploring CIs, and a formulation for non-adiabatic coupling matrix elements (NACMEs) exists [18]; however, actual implementation has not been achieved.

In 2001, Angeli et al. proposed another variant of MRPT methods, $n$ electron valence state second-order perturbation theory (NEVPT2) [19, 20, 21], using uncontracted and two internal contraction schemes. Recently, analytic derivatives of the partially (PC) $[22,23]$ and strongly contracted (SC) [23] NEVPT2 method have been realized, and both pilot calculations presented promising results. There are two known advantages of NEVPT2 over CASPT2: exact size consistency and the lack of an intruder state problem. Although the former is trivial because the internally contracted CASPT2 is nearly size-consistent, the latter is an appealing feature because NEVPT2 does not require any a posteriori parameters. Additionally, no large linear equations have been solved for the standard NEVPT2; thus, it is computationally advantageous.

Recently, Park developed [24] analytic gradients and derivative coupling vectors for the quasidegenerate extension of the PC-NEVPT2 method, namely QD-PC-NEVPT2. In this Letter, analytic gradients and interstate coupling 
vectors of the QD-PC-NEVPT2 and QD-SC-NEVPT2 methods [25] were developed, and the methodologies have been applied to locating CIs in benzene. The results have been compared with CASSCF, XMS-CASPT2, and spin-flip time-dependent density functional theory (SF-TD-DFT) [26] calculations.

\section{Methodology}

\subsection{Overview of the QD-NEVPT2 method}

The single-state or state-specific (SS) NEVPT2 energy may be expressed as a sum of the CASSCF and the second-order perturbation energy:

$$
E_{S}^{\mathrm{SS}-\mathrm{NEVPT} 2}=E_{S}^{\mathrm{CAS}}+E_{S}^{\mathrm{PT} 2}
$$

where the second-order perturbation correction is given by

$$
E_{S}^{\mathrm{PT} 2}=E_{S}^{(0)}+E_{S}^{(-1)}+E_{S}^{(+1)}+E_{S}^{(-2)}+E_{S}^{(+2)}+E_{S}^{(0)^{\prime}}+E_{S}^{(-1)^{\prime}}+E_{S}^{(+1)^{\prime}},
$$

consisting of eight terms which correspond to eight unique excitation classes defined in Ref. [21]. Each term is computed independently because the coupling between different excitation classes is neglected in NEVPT2. This kind of internally contracted SS-MRPT methods cannot correctly describe the avoided crossing region of the ionic and neutral states of $\mathrm{LiF}[25,27]$ and results in a (wrong) double crossing. As a solution to this problem, several internal states (usually the states averaged in the reference CASSCF calculation) are allowed to couple at the second-order through an effective Hamiltonian in the quasidegenerate approach. A similar quasidegenerate approach has been employed in the multiconfiguration quasidegenerate second-order perturbation theory (MCQDPT2) [28].

QD-NEVPT2 [25] is a multistate extension of SS-NEVPT2, as in the case of MS-CASPT2. In QD-NEVPT2, the effective Hamiltonian matrix is first constructed:

$$
\bar{H}_{S T}^{\mathrm{eff}}=\delta_{S T} E_{S}^{\mathrm{CAS}}+\frac{1}{2}\left(H_{S T}+H_{T S}\right)
$$

where

$$
H_{S T}=\left\langle\Psi_{S}^{(0)}|\hat{H}| \Psi_{T}^{(1)}\right\rangle,
$$

where $\Psi_{S}^{(0)}$ and $\Psi_{S}^{(1)}$ are the zeroth-order and first-order correction to wavefunctions of the state $S$, respectively. The diagonal elements of the effective Hamiltonian correspond to the SS-NEVPT2 energies [Eq. (1)]. Diagonalization of the symmetrized effective Hamiltonian, $\bar{H}_{S T}^{\text {eff }}$, leads to the 
QD-NEVPT2 energy. Note that the effective Hamiltonian matrix is symmetrized in this study, whereas the original formulation [25] employed the non-Hermitian form. This is not only due to technical issues relevant to the difficulty of diagonalizing non-Hermitian matrices. Symmetrization of the effective Hamiltonian is necessary to produce the correct $3 N_{\text {atom }}-8$ dimensional intersection space [29].

There are two canonicalization schemes for MRPT methods using SAMCSCF or SA-CASSCF references: SS- and SA-Fock operators [30]. The two schemes differ in the definition of the orbital energy of the doubly occupied and virtual orbitals. The SS canonicalization employs SS density matrix to obtain the orbital energy, whereas the SA one employs a SA density matrix, which is the usual choice when SA-MCSCF or SA-CASSCF wavefunctions are obtained. Although the former definition may be employed in the initial QD-NEVPT2 development [25] and is sometimes thought to be more accurate, the latter definition was adopted in this study as in the previous study [22] as well as in the work by Park [24] for simplicity. The difference between the two canonicalization schemes is not significant [30]. Apart from the choice of the canonicalization, both the QD-PC-NEVPT2 and QD-SC-NEVPT2 methods are still not fully invariant $[14,31]$ in the first-order interacting space, but the expected degradation is rather small compared with MS-CASPT2. Indeed, QD-PC-NEVPT2 can correctly describe CI points [24, 29] as can XMS-CASPT2 and the extended MCQDPT2 (XMCQDPT2) by Nakano and Granovsky [14, 28].

\subsection{Analytic derivatives of $Q D-N E V P T 2$}

The QD-NEVPT2 energy may be written as

$$
E_{S}^{\mathrm{QD}-\mathrm{NEVPT} 2}=\sum_{T U}^{\Omega} \mathcal{C}_{T S} \mathcal{C}_{U S} \bar{H}_{T U}^{\mathrm{eff}}
$$

where $\mathcal{C}_{S T}$ is a unitary matrix that diagonalizes the effective Hamiltonian:

$$
\sum_{T}^{\Omega} \bar{H}_{S T}^{\mathrm{eff}} \mathcal{C}_{T U}=\mathcal{C}_{S U} E_{U}^{\mathrm{QD}-\mathrm{NEVPT} 2}
$$

where $\Omega$ represents the internal state. 
The first-order derivative of the QD-NEVPT2 energy with respect to the derivative parameter, $\alpha$, is

$$
\frac{\mathrm{d} E_{S}^{\mathrm{QD}-\mathrm{NEVPT} 2}}{\mathrm{~d} \alpha}=\sum_{T U}^{\Omega} \mathcal{C}_{T S} \mathcal{C}_{U S} \frac{\mathrm{d} \bar{H}_{T U}^{\mathrm{eff}}}{\mathrm{d} \alpha}
$$

At the first-order derivative, the derivative of $\mathcal{C}_{S T}$ disappears because it is unitary:

$$
\sum_{S}^{\Omega}\left(\frac{\mathrm{d} \mathcal{C}_{T S}}{\mathrm{~d} \alpha} \mathcal{C}_{U S}+\mathcal{C}_{T S} \frac{\mathrm{d} \mathcal{C}_{U S}}{\mathrm{~d} \alpha}\right)=0
$$

Thus, the present implementation is therefore applicable only to the symmetric effective Hamiltonian [Eq. (3)].

The derivative of $\bar{H}_{S T}^{\text {eff }}$ can be computed as in previous studies [22, 23, 24]. In particular, the diagonal elements are equivalent to the SS-NEVPT2 energy. The off-diagonal elements are also similar; however, an additional computation is required: the evaluation of the derivatives of the transition reduced density matrices (TRDMs) for up to three particles

$$
\sum_{I J} c_{I}^{S} c_{J}^{T}\left\langle I\left|a_{a}^{\dagger} a_{b}^{\dagger} a_{c}^{\dagger} a_{f} a_{e} a_{d}\right| J\right\rangle
$$

where $c_{I}^{S}$ is the configuration coefficient for state $S$. The partial derivatives of the TRDMs, which are relevant to Eqs. (12) and (13), are evaluated using the resolution of the identity technique [32].

Eventually, as in the previous study [22], the derivative of the QD-NEVPT2 energy may be written as

$$
\begin{aligned}
\frac{\mathrm{d} E_{S}^{\mathrm{QD}-N E V P T} 2}{\mathrm{~d} \alpha} & =\sum_{\mu \nu} d_{\mu \nu}^{S} \frac{\mathrm{d} h_{\mu \nu}}{\mathrm{d} \alpha}-\sum_{\mu \nu} X_{\mu \nu}^{S} \frac{\mathrm{d} S_{\mu \nu}}{\mathrm{d} \alpha}+\sum_{\mu \nu \rho \sigma} D_{\mu \nu \rho \sigma}^{S} \frac{\mathrm{d}(\mu \nu \mid \rho \sigma)}{\mathrm{d} \alpha} \\
& +\sum_{p q}^{\text {indep }} \mathcal{L}_{p q}^{\mathrm{o}} \widetilde{U}_{p q}^{\alpha}+\sum_{T}^{\Omega} \sum_{I}^{N} \mathcal{L}_{T I}^{\mathrm{c}} V_{T I}^{\alpha}+\sum_{T<U}^{\Omega} \mathcal{L}_{T U}^{\mathrm{s}} W_{T U}^{\alpha}
\end{aligned}
$$

where $d_{\mu \nu}^{S}, X_{\mu \nu}^{S}$, and $D_{\mu \nu \rho \sigma}^{S}$ are unrelaxed one-body, energy-weighted, and two-body density matrices in the atomic orbital basis, respectively. The last three terms are relevant to the derivative of the wavefunction parameters 
(molecular orbital and configuration coefficients), and they are written as partial derivatives of the total energy:

$$
\begin{gathered}
\mathcal{L}_{p q}^{\mathrm{o}}=\left(1-\hat{\tau}_{p q}\right) \sum_{\mu} C_{\mu p} \frac{\partial E^{\mathrm{QD}-\mathrm{NEVPT} 2}}{\partial C_{\mu q}}, \\
\mathcal{L}_{S I}^{\mathrm{c}}=\sum_{J} M_{I J} \frac{\partial E^{\mathrm{QD}-\mathrm{NEVPT} 2}}{\partial c_{J}^{S}}
\end{gathered}
$$

and

$$
\mathcal{L}_{S T}^{\mathrm{s}}=\left(1-\hat{\tau}_{S T}\right) \sum_{I} \frac{\partial E^{\mathrm{QD}-\mathrm{NEVPT} 2}}{\partial c_{I}^{S}} c_{I}^{T} .
$$

Operator $\hat{\tau}_{p q}$ commutes indices $p$ and $q, C_{\mu i}$ is the molecular orbital coefficient, and projection $M_{I J}$ was defined in an earlier study [33]. Once transformed above, the Z-vector equation based on the MCSCF wavefunction can be solved, and the solution is contracted to obtain the final analytic gradient. More details on SS-NEVPT2 are presented in Refs. [22] and [23], and those for QD-PC-NEVPT2 are in Ref. [24].

\subsection{Interstate Coupling Vectors}

At the CI region, the degeneracies of the two adiabatic states are lifted along the gradient difference and interstate coupling vectors, which then define the branching plane. Although it is currently possible to locate CIs without interstate coupling vectors by branching plane updating [34] or penalty function $[35,36]$ methods, they should aid a smoother convergence if the two vectors are available. The gradient difference vector for states $S$ and $T$ is simply obtained by

$$
g_{\alpha}^{S T}=\frac{\mathrm{d} E_{T}^{\mathrm{QD}-\mathrm{NEVPT} 2}}{\mathrm{~d} \alpha}-\frac{\mathrm{d} E_{S}^{\mathrm{QD}-\mathrm{NEVPT} 2}}{\mathrm{~d} \alpha} ;
$$

Thus, it can be computed using the method in the previous subsection. Interstate coupling vectors can also be computed as the first-order derivative of off-diagonal elements of the diagonalized effective Hamiltonian matrix. Thus, they are expressed by

$$
h_{\alpha}^{S T}=\frac{1}{E_{T}^{\mathrm{QD}-\mathrm{NEVPT} 2}-E_{S}^{\mathrm{QD}-\mathrm{NEVPT} 2}} \sum_{U V} \mathcal{C}_{U S} \mathcal{C}_{V T} \frac{\mathrm{d} \bar{H}_{U V}^{\mathrm{eff}}}{\mathrm{d} \alpha},
$$


and this is very similar to the first-order derivative expression of the QDNEVPT2 energy [Eq. (7)]; therefore, it can be computed using a similar algorithm for the derivative of the QD-NEVPT2 energy presented in the previous subsection. The extension to full derivative coupling vectors or NACMEs should be straightforward, but it was not developed in this study because the interstate coupling was sufficient to locate the CIs.

\section{Computational details}

All CASSCF, QD-PC-NEVPT2, QD-SC-NEVPT2, and SF-TD-DFT calculations were performed using a locally modified version of GAMESS-US [37], whereas the (fully internally contracted) XMS-CASPT2 calculations were performed using BAGEL $[38,39]$. The frozen core approximation was applied to all post-CASSCF calculations. No symmetry constraints were applied.

CASSCF, QD-PC-NEVPT2, QD-SC-NEVPT2, XMS-CASPT2, and SFTD-DFT calculations were performed for benzene using the cc-pVTZ basis

set. The active space consisted of six electrons in six orbitals (6e, 6o), and two states were averaged with equal weight. In the SF-TD-DFT [40] calculation, restricted open-shell triplet wavefunctions were employed as a reference, and the BHHLYP functional [41, 42] was employed as in Ref. [43]. An imaginary level shift [44] of $0.2 \mathrm{i}$ and density fitting were applied in the XMS-CASPT2 calculations.

The CI search was performed using the gradient projection method [45]. Some preliminary calculations indicated that the MECIs located by the branching plane updating method by Maeda et al. [34] agree with those located using the gradient projection method using analytic interstate coupling vectors. All the optimized coordinates are provided in the Supporting Information.

\section{Results and discussion}

In this section, QD-PC-NEVPT2, QD-SC-NEVPT2, and XMS-CASPT2 are simply written as PC-NEVPT2, SC-NEVPT2, and CASPT2, respectively, for simplicity. NEVPT2 without the prefix collectively stands for PC-NEVPT2 and SC-NEVPT2. 


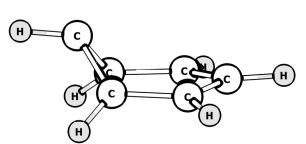

1: $0.00 \mathrm{eV}$

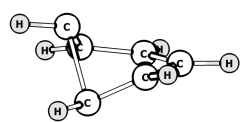

5: $2.24 \mathrm{eV}$
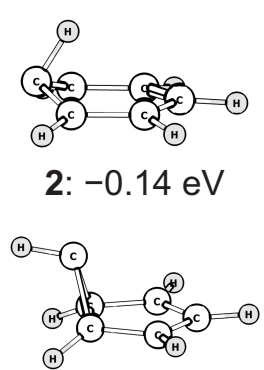

6: $0.10 \mathrm{eV}$

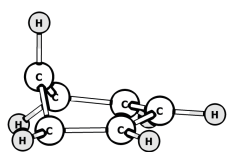

3: $0.17 \mathrm{eV}$

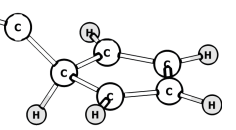

7: $1.39 \mathrm{eV}$

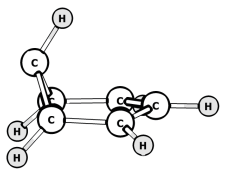

4: $0.55 \mathrm{eV}$

(11)

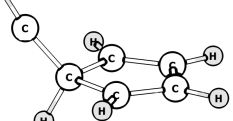

8: $1.52 \mathrm{eV}$

Figure 1: Optimized structures of the eight MECIs of benzene located using PC-NEVPT2 in this study. The energies are relative to $\mathbf{1}$ at PC-NEVPT2.

\subsection{CIs of benzene}

As a pilot application of QD-NEVPT2, CIs located by Harabuchi et al. at the SF-TD-DFT/6-31G(d) level of theory [43] were relocated using the CASSCF, PC-NEVPT2, SC-NEVPT2, CASPT2, and SF-TD-DFT methods using the cc-pVTZ basis set. In Table 1, the energies of eight MECIs relative to the lowest MECI 1 at SF-TD-DFT are summarized, and the optimized structures with PC-NEVPT2 are shown in Figure 1. The numbering is consistent with that in Ref. [43]. The computed excitation energies for the global minimum $\left(D_{6 \mathrm{~h}}\right)$ structure were 4.98, 5.35, 4.87, and $5.33 \mathrm{eV}$ according to the CASSCF, PC-NEVPT2, CASPT2, and SF-TD-DFT levels of theory, respectively. The experimental excitation energy was $4.9 \mathrm{eV} \mathrm{[46];} \mathrm{thus,} \mathrm{the}$ one predicted with CASPT2 agreed quite well with the experimental result, whereas the PC-NEVPT2 and SF-TD-DFT overestimated the result. The global minimum could not be located using the SC-NEVPT2 method because of the numerical instability observed previously with SS-SC-NEVPT2 [23]. This instability is due to the non-invariance of the perturbation energy under rotations within doubly occupied and virtual orbitals, and this character necessitates division by the difference in orbital energies, which becomes trivial for highly symmetric molecules, to obtain an unrelaxed density matrix. No such instability was detected during the CI search.

Compared with CASSCF and the three MRPT methods (PC-NEVPT2, SC-NEVPT2, and CASPT2), clearly, the difference can be more than $0.7 \mathrm{eV}$, which was found for 2 . Surprisingly, the performance of SF-TD-DFT was found to be overall better than CASSCF. Although it also gave a somewhat 
Table 1: Energies relative to the lowest MECI 1 at SF-TD-DFT (unit in eV).

\begin{tabular}{lccccc}
\hline & CASSCF & PC-NEVPT2 & SC-NEVPT2 & CASPT2 & SF-TD-DFT \\
\hline $\mathrm{S}_{0}$ at $D_{6 \mathrm{~h}}$ & -5.57 & -5.30 & $-{ }^{b}$ & -4.95 & -5.64 \\
$\mathrm{~S}_{1}$ at $D_{6 \mathrm{~h}}$ & -0.57 & 0.06 & $-{ }^{b}$ & -0.09 & -0.31 \\
$\mathbf{1}$ & 0.00 & 0.00 & 0.00 & 0.00 & 0.00 \\
$\mathbf{2}$ & 0.79 & -0.14 & -0.12 & 0.06 & 0.09 \\
$\mathbf{3}$ & 0.35 & 0.17 & 0.17 & 0.17 & 0.11 \\
$\mathbf{4}$ & 0.28 & 0.55 & 0.54 & 0.50 & 0.67 \\
$\mathbf{5}$ & $2.46^{a}$ & $2.24^{a}$ & $2.27^{a}$ & 2.23 & 2.14 \\
$\mathbf{6}$ & 0.52 & 0.10 & 0.11 & 0.19 & 0.24 \\
$\mathbf{7}$ & 0.77 & 1.39 & 1.37 & 1.39 & 0.96 \\
$\mathbf{8}$ & 0.89 & 1.52 & 1.49 & 1.53 & 1.04 \\
\hline
\end{tabular}

${ }^{a}$ Saddle point optimizations were performed. ${ }^{b}$ SC-NEVPT2 could not locate the $D_{6 \mathrm{~h}}$ structure.

large difference of $0.4-0.5 \mathrm{eV}$ in the relative energy for $\mathbf{7}$ and $\mathbf{8}$, it predicted a similar energy to NEVPT2 and CASPT2 for the other MECIs. The difference between NEVPT2 and CASPT2 was overall small, and that for $\mathbf{3}$ to $\mathbf{8}$ was less than $0.1 \mathrm{eV}$.

Interestingly, PC-NEVPT2 and SC-NEVPT2 predicted that $\mathbf{2}$ was the most stable CI among the eight CIs, whereas the other three methods showed that $\mathbf{1}$ was the most stable. To verify the relative energy between $\mathbf{1}$ and $\mathbf{2}$, single-point calculations were performed for the $\mathbf{1}$ and $\mathbf{2}$ structures obtained with PC-NEVPT2 at the fully internally contracted MRCI with the sizeconsistency correction (FIC-MRCI+Q) [47], as implemented in ORCA [48] Version 4.2.1, although this method is not well suited for describing CI regions $[29,47]$. The FIC-MRCI+Q result indicated that 2 was $0.06 \mathrm{eV}$ less stable, and this supports the CASPT2 and SF-TD-DFT result. Although the difference between NEVPT2 and CASPT2 is especially significant for $\mathbf{2}$, it was less than $0.2 \mathrm{eV}$, which is the expected accuracy of MRPT methods. In addition, considering that the relative energy of $\mathbf{2}$ is significantly lowered after perturbative corrections, the quality of the zeroth-order wavefunction may be somewhat poor.

Locating the highest energy CI 5 was not straightforward with GAMESS- 
US even at the CASSCF level. This was because $\mathbf{5}$ was not a minimum in the intersection space; in this study, this was verified via vibrational frequency analysis using Hessian obtained by numerical differentiation of the intersection gradient:

$$
\mathbf{g}^{\mathrm{IS}}=\mathbf{P g}^{\text {mean }},
$$

where $\mathbf{g}^{\text {mean }}$ is the average of the gradients at the two states and $\mathbf{P}$ is the projection matrix

$$
\mathbf{P}=\mathbf{1}-\mathbf{g}^{\prime} \mathbf{g}^{\prime T}-\mathbf{h}^{\prime} \mathbf{h}^{\prime T}
$$

where $\mathbf{g}^{\prime}$ and $\mathbf{h}^{\prime}$ are the orthonormalized gradient difference and interstate coupling vectors, respectively, and the superscript of $\mathrm{T}$ stands for the transpose of the vector. The gradient difference and interstate coupling vectors were further projected out from the Hessian. Indeed, the vibrational frequency analysis using this approximate Hessian after geometry optimization revealed that it is a saddle point whose imaginary mode has a frequency of $-523 \mathrm{~cm}^{-1}$ at PC-NEVPT2. Geometry optimizations using displaced geometries along the imaginary mode indicated that the saddle point should connect mirror isomers of $\mathbf{6}$. Locating $\mathbf{5}$ at CASPT2 with BAGEL and SFTD-DFT with GAMESS-US did not exhibit this difficulty. For quantitative discussions, intersection Hessian and intrinsic reaction coordinate [49] calculations must be performed.

A comparison of the geometries is summarized in Table 2. First, the root-mean-square differences (RMSDs) for each structure were computed for all possible combinations (see Tables S1-S8 in the Supporting Information). Then, the RMSDs thus obtained were further calculated and are summarized in Table 2. The RMSD between PC-NEVPT2 and SC-NEVPT2 was only $0.004 \AA$; the difference in the structures was also trivial as in the case of the relative energy. The largest difference of $0.010 \AA$ was found for 8 (Table $\mathrm{S} 8$ in the Supporting Information). One hydrogen atom was rather floppy, and the movement of the hydrogen atom formed a shallow potential energy surface. The same applies to SF-TD-DFT, and the RMSDs against the MRPT methods were somewhat large $(\sim 0.14 \AA)$. Notably, the RMSDs for the CASSCF-MRPTs (PC-NEVPT2, SC-NEVPT2, and CASPT2) were greater than $0.1 \AA$, whereas that for NEVPT2-CASPT2 was approximately four times smaller $(\sim 0.025 \AA)$. The largest difference between CASSCF and the MRPTs was found for 4 . A comparison of the optimized structures for CASSCF and PC-NEVPT2 is presented in Figure 2 in which the planar part is maximally superposed to clarify the difference. Clearly, the orientations 
Table 2: RMSDs (in $\AA$ ) of RMSDs for the eight CIs.

\begin{tabular}{lccccc}
\hline & CASSCF & PC-NEVPT2 & SC-NEVPT2 & CASPT2 & SF-TD-DFT \\
\hline CASSCF & - & & & & \\
PC-NEVPT2 & 0.117 & - & & & \\
SC-NEVPT2 & 0.117 & 0.004 & - & & \\
CASPT2 & 0.103 & 0.025 & 0.024 & - & \\
SF-TD-DFT & 0.111 & 0.060 & 0.059 & 0.063 & - \\
\hline
\end{tabular}

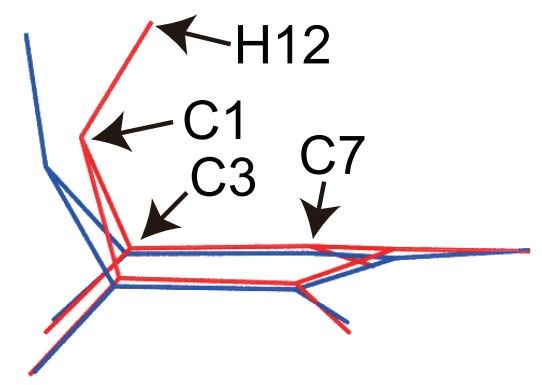

Figure 2: Superposed structures of 4 optimized at the CASSCF (in blue) and PC-NEVPT2 (in red) levels of theory.

of $\mathrm{C} 1$ and $\mathrm{H} 12$ were significantly different. To quantify, the dihedral angle of $\mathrm{H} 12-\mathrm{C} 1-\mathrm{C} 3-\mathrm{C} 7$ as defined herein was $-78.2^{\circ}$ and $-35.0^{\circ}$ for $\mathrm{CASSCF}$ and $\mathrm{PC}-\mathrm{NEVPT}$, respectively. This difference in geometry is reflected in the relative energy of $\mathbf{4}$, which was only $0.28 \mathrm{eV}$ above $\mathbf{1}$ for CASSCF, whereas it was $\sim 0.50 \mathrm{eV}$ for the MRPT methods. These results highlight the importance of adding dynamic electron correlation for obtaining better structural parameters.

Considering that the relative energies obtained with SF-TD-DFT are closer to those of NEVPT2 and CASPT2 than to that of CASSCF, SFTD-DFT is a reasonable method to screen MECI candidates and even for practical calculations. In addition to the relative energy, the RMSDs of the geometries for MRPTs-SF-TD-DFT (Table 2) are smaller than CASSCF: approximately half of those with CASSCF and twice of those with MRPT comparisons. As long as the wavefunction obtained with SF-TD-DFT is 
Table 3: $\left\langle S^{2}\right\rangle$ values for the SF-TD-DFT results; " $\mathrm{S}_{0}$ " and " $\mathrm{S}_{1}$ " are denoted only formally based on the absolute energy and do not reflect the excitation character and formal $\left\langle S^{2}\right\rangle$.

\begin{tabular}{lcc}
\hline & $\mathrm{S}_{0}$ & $\mathrm{~S}_{1}$ \\
\hline$D_{6 \mathrm{~h}}$ & 0.011 & 1.001 \\
$\mathbf{1}$ & 0.418 & 0.437 \\
$\mathbf{2}$ & 0.618 & 0.362 \\
$\mathbf{3}$ & 0.942 & 0.218 \\
$\mathbf{4}$ & 0.758 & 0.571 \\
$\mathbf{5}$ & 0.088 & 0.500 \\
$\mathbf{6}$ & 0.684 & 0.186 \\
$\mathbf{7}$ & 0.013 & 0.013 \\
$\mathbf{8}$ & 0.093 & 0.010 \\
\hline
\end{tabular}

valid, SF-TD-DFT is a reasonable choice for locating MECIs. Here, the $\left\langle S^{2}\right\rangle$ values at the two states are summarized in Table 3 . These values are similar to those at the SF-TD-DFT/6-31G(d) level of theory obtained by Harabuchi [43]. The highest $\left\langle S^{2}\right\rangle$ value found in this study was greater than one (for $\mathrm{S}_{1}$ at the global minimum structure). It is clearly too large to identify as the singlet state. Despite the better agreement of the SF-TD-DFT energy and geometry with MRPT methods than with CASSCF, as presented in this study, the quantity of the wavefunction should be carefully assessed.

\section{Conclusions}

In this study, analytic derivatives of QD-PC-NEVPT2 and QD-SC-NEVPT2 methods were developed and implemented in a local version of GAMESS-US. The methodology was applied to locating CIs in a typical system: benzene. The results from QD-PC-NEVPT2 and QD-SC-NEVPT2 were quite similar each other, and the result from QD-NEVPT2 was overall similar to that of XMS-CASPT2. The results presented in this Letter also highlight the importance of adding dynamical electron correlation. SF-TD-DFT overall demonstrated better performance than CASSCF for relative energies and optimized structures, but the spin contaminations were significant. Additionally, the character of the stationary point in the intersection space should be verified by performing vibrational frequency analysis. 


\section{Acknowledgements}

This work was partially supported by JSPS KAKENHI Grant No. 17K14436 and $19 \mathrm{H} 02682$.

\section{References}

[1] D. R. Yarkony, Diabolical conical intersections, Rev. Mod. Phys. 68 (1996) 985-1013 (1996). doi:10.1103/RevModPhys.68.985.

[2] D. R. Yarkony, Conical intersections: The new conventional wisdom, J. Phys. Chem. A 105 (26) (2001) 6277-6293 (2001). doi:10.1021/jp003731u.

[3] W. Domcke, D. R. Yarkony, Role of conical intersections in molecular spectroscopy and photoinduced chemical dynamics, Annu. Rev. Phys. Chem. 63 (1) (2012) 325-352 (2012). doi:10.1146/annurev-physchem032210-103522.

[4] B. O. Roos, R. Lindh, P. A. Malmqvist, V. Veryazov, P.-O. Widmark (Eds.), Multiconfigurational Quantum Chemistry, John Wiley \& Sons, Ltd., Hoboken, New Jersey, 2016 (2016).

[5] M. Musiał, A. Perera, R. J. Bartlett, Multireference coupled-cluster theory: The easy way, J. Chem. Phys. 134 (11) (2011) 114108 (2011). doi:10.1063/1.3567115.

[6] D. I. Lyakh, M. Musiał, V. F. Lotrich, R. J. Bartlett, Multireference nature of chemistry: The coupled-cluster view, Chem. Rev. 112 (1) (2012) 182-243 (2012). doi:10.1021/cr2001417.

[7] A. Köhn, M. Hanauer, L. A. Mück, T.-C. Jagau, J. Gauss, State-specific multireference coupled-cluster theory, WIREs Comput. Mol. Sci. 3 (2) (2013) 176-197 (2013). doi:10.1002/wcms.1120.

[8] P. G. Szalay, T. Müller, G. Gidofalvi, H. Lischka, R. Shepard, Multiconfiguration self-consistent field and multireference configuration interaction methods and applications, Chem. Rev. 112 (1) (2012) 108-181 (2012). doi:10.1021/cr200137a. 
[9] J. W. Park, R. Al-Saadon, M. K. MacLeod, T. Shiozaki, B. Vlaisavljevich, Multireference electron correlation methods: Journeys along potential energy surfaces, arXiv (2019) 1911.06836 (2019).

[10] B. O. Roos, P. Linse, P. E. Siegbahn, M. R. Blomberg, A simple method for the evaluation of the second-order-perturbation energy from external double-excitations with a casscf reference wavefunction, Chem. Phys. 66 (1) (1982) 197 - 207 (1982). doi:https://doi.org/10.1016/03010104(82)88019-1.

[11] K. Andersson, P. A. Malmqvist, B. O. Roos, A. J. Sadlej, K. Wolinski, Second-order perturbation theory with a casscf reference function, J. Phys. Chem. 94 (14) (1990) 5483-5488 (1990). doi:10.1021/j100377a012.

[12] K. Andersson, P. Malmqvist, B. O. Roos, Second-order perturbation theory with a complete active space self-consistent field reference function, J. Chem. Phys. 96 (2) (1992) 1218-1226 (1992). doi:10.1063/1.462209.

[13] J. W. Park, T. Shiozaki, Analytical derivative coupling for multistate caspt2 theory, J. Chem. Theory Comput. 13 (6) (2017) 2561-2570 (2017). doi:10.1021/acs.jctc.7b00018.

[14] A. A. Granovsky, Extended multi-configuration quasi-degenerate perturbation theory: The new approach to multi-state multi-reference perturbation theory, J. Chem. Phys. 134 (21) (2011) 214113 (2011). doi:10.1063/1.3596699.

[15] T. Shiozaki, W. Győrffy, P. Celani, H.-J. Werner, Communication: Extended multi-state complete active space second-order perturbation theory: Energy and nuclear gradients, J. Chem. Phys. 135 (8) (2011) 081106 (2011). doi:10.1063/1.3633329.

[16] J. W. Park, T. Shiozaki, On-the-fly caspt2 surface-hopping dynamics, J. Chem. Theory Comput. 13 (8) (2017) 3676-3683 (2017). doi:10.1021/acs.jctc.7b00559.

[17] Y. G. Khait, J. Song, M. R. Hoffmann, Explication and revision of generalized van vleck perturbation theory for molecular electronic structure, J. Chem. Phys. 117 (9) (2002) 4133-4145 (2002). doi:10.1063/1.1497642. 
[18] Y. G. Khait, D. Theis, M. R. Hoffmann, Nonadiabatic coupling terms for the gvvpt2 variant of multireference perturbation theory, Chem. Phys. 401 (2012) 88 - 94 (2012). doi:https://doi.org/10.1016/j.chemphys.2011.09.014.

[19] C. Angeli, R. Cimiraglia, S. Evangelisti, T. Leininger, J.-P. Malrieu, Introduction of $n$-electron valence states for multireference perturbation theory, J. Chem. Phys. 114 (23) (2001) 10252-10264 (2001). doi:10.1063/1.1361246.

[20] C. Angeli, R. Cimiraglia, J.-P. Malrieu, $n$-electron valence state perturbation theory: a fast implementation of the strongly contracted variant, Chem. Phys. Lett. 350 (3) (2001) 297 - 305 (2001). doi:https://doi.org/10.1016/S0009-2614(01)01303-3.

[21] C. Angeli, R. Cimiraglia, J.-P. Malrieu, $n$-electron valence state perturbation theory: A spinless formulation and an efficient implementation of the strongly contracted and of the partially contracted variants, J. Chem. Phys. 117 (20) (2002) 9138-9153 (2002). doi:10.1063/1.1515317.

[22] Y. Nishimoto, Analytic first-order derivatives of partially contracted nelectron valence state second-order perturbation theory (pc-nevpt2), J. Chem. Phys. 151 (11) (2019) 114103 (2019). doi:10.1063/1.5115819.

[23] J. W. Park, Analytical gradient theory for strongly contracted (sc) and partially contracted (pc) n-electron valence state perturbation theory (nevpt2), J. Chem. Theory Comput. 15 (10) (2019) 5417-5425 (2019). doi:10.1021/acs.jctc.9b00762.

[24] J. W. Park, Analytical gradient theory for quasidegenerate n-electron valence state perturbation theory (qd-nevpt2), J. Chem. Theory Comput. 16 (1) (2020) 326-339 (2020). doi:10.1021/acs.jctc.9b00919.

[25] C. Angeli, S. Borini, M. Cestari, R. Cimiraglia, A quasidegenerate formulation of the second order $n$-electron valence state perturbation theory approach, J. Chem. Phys. 121 (9) (2004) 4043-4049 (2004). doi:10.1063/1.1778711.

[26] Y. Shao, M. Head-Gordon, A. I. Krylov, The spin-flip approach within time-dependent density functional theory: Theory and applica- 
tions to diradicals, J. Chem. Phys. 118 (11) (2003) 4807-4818 (2003). doi:10.1063/1.1545679.

[27] J. Finley, P. Åke Malmqvist, B. O. Roos, L. Serrano-Andrés, The multistate caspt2 method, Chem. Phys. Lett. 288 (2) (1998) 299 - 306 (1998). doi:https://doi.org/10.1016/S0009-2614(98)00252-8.

[28] H. Nakano, Quasidegenerate perturbation theory with multiconfigurational self-consistent-field reference functions, J. Chem. Phys. 99 (10) (1993) 7983-7992 (1993). doi:10.1063/1.465674.

[29] S. Gozem, F. Melaccio, A. Valentini, M. Filatov, M. Huix-Rotllant, N. Ferré, L. M. Frutos, C. Angeli, A. I. Krylov, A. A. Granovsky, R. Lindh, M. Olivucci, Shape of multireference, equation-of-motion coupled-cluster, and density functional theory potential energy surfaces at a conical intersection, J. Chem. Theory Comput. 10 (8) (2014) 30743084 (2014). doi:10.1021/ct500154k.

[30] I. Schapiro, K. Sivalingam, F. Neese, Assessment of n-electron valence state perturbation theory for vertical excitation energies, J. Chem. Theory Comput. 9 (8) (2013) 3567-3580 (2013). doi:10.1021/ct400136y.

[31] On the non-invariance of qd-nevpt2 theory http://classic.chem.msu.su/gran/gamess/qdnevpt2-non-invariance.pdf (accessed on january 15, 2020).

[32] P. J. Knowles, H.-J. Werner, An efficient method for the evaluation of coupling coefficients in configuration interaction calculations, Chem. Phys. Lett. 145 (6) (1988) 514 - 522 (1988). doi:https://doi.org/10.1016/0009-2614(88)87412-8.

[33] K. L. Bak, J. Boatz, J. Simons, First-order geometrical response equations for state-averaged multiconfigurational self-consistent field (samcscf) wave functions, Int. J. Quantum Chem. 40 (3) (1991) 361-378 (1991). doi:10.1002/qua.560400308.

[34] S. Maeda, K. Ohno, K. Morokuma, Updated branching plane for finding conical intersections without coupling derivative vectors, J. Chem. Theory Comput. 6 (5) (2010) 1538-1545 (2010). doi:10.1021/ct1000268. 
[35] B. G. Levine, C. Ko, J. Quenneville, T. J. Martínez, Conical intersections and double excitations in time-dependent density functional theory, Mol. Phys. 104 (5-7) (2006) 1039-1051 (2006). doi:10.1080/00268970500417762.

[36] B. G. Levine, J. D. Coe, T. J. Martínez, Optimizing conical intersections without derivative coupling vectors: Application to multistate multireference second-order perturbation theory (ms-caspt2), J. Phys. Chem. B 112 (2) (2008) 405-413 (2008). doi:10.1021/jp0761618.

[37] M. W. Schmidt, K. K. Baldridge, J. A. Boatz, S. T. Elbert, M. S. Gordon, J. H. Jensen, S. Koseki, N. Matsunaga, K. A. Nguyen, S. Su, T. L. Windus, M. Dupuis, J. A. Montgomery, General atomic and molecular electronic structure system, J. Comput. Chem. 14 (1993) 1347-1363 (1993).

[38] Bagel, brilliantly advanced general electronic-structure library. http://www.nubakery.org under the gnu general public license., (accessed on june 20, 2019).

[39] T. Shiozaki, Bagel: Brilliantly advanced general electronic-structure library, WIREs Comput. Mol. Sci. 8 (1) (2018) e1331 (2018). doi:10.1002/wcms.1331.

[40] N. Minezawa, M. S. Gordon, Optimizing conical intersections by spin flip density functional theory: Application to ethylene, J. Phys. Chem. A 113 (46) (2009) 12749-12753 (2009). doi:10.1021/jp908032x.

[41] A. D. Becke, Density-functional exchange-energy approximation with correct asymptotic behavior, Phys. Rev. A 38 (1988) 3098-3100 (Sep 1988). doi:10.1103/PhysRevA.38.3098.

URL http://link.aps.org/doi/10.1103/PhysRevA.38.3098

[42] C. Lee, W. Yang, R. G. Parr, Development of the colle-salvetti correlation-energy formula into a functional of the electron density, Phys. Rev. B 37 (1988) 785-789 (Jan 1988). doi:10.1103/PhysRevB.37.785.

URL http://link.aps.org/doi/10.1103/PhysRevB.37.785

[43] Y. Harabuchi, T. Taketsugu, S. Maeda, Combined gradient projection/single component artificial force induced reaction (gp/sc-afir) 
method for an efficient search of minimum energy conical intersection (meci) geometries, Chem. Phys. Lett. 674 (2017) 141 - 145 (2017). doi:https://doi.org/10.1016/j.cplett.2017.02.069.

[44] J. W. Park, R. Al-Saadon, N. E. Strand, T. Shiozaki, Imaginary shift in caspt2 nuclear gradient and derivative coupling theory, J. Chem. Theory Comput. 15 (7) (2019) 4088-4098 (2019). doi:10.1021/acs.jctc.9b00368.

[45] M. J. Bearpark, M. A. Robb, H. B. Schlegel, A direct method for the location of the lowest energy point on a potential surface crossing, Chem. Phys. Lett. 223 (3) (1994) 269 - 274 (1994). doi:https://doi.org/10.1016/0009-2614(94)00433-1.

[46] E. N. Lassettre, A. Skerbele, M. A. Dillon, K. J. Ross, High- resolution study of electron- impact spectra at kinetic energies between 33 and $100 \mathrm{ev}$ and scattering angles to $16^{\circ}$, J. Chem. Phys. 48 (11) (1968) 5066-5096 (1968). doi:10.1063/1.1668178.

[47] K. Sivalingam, M. Krupicka, A. A. Auer, F. Neese, Comparison of fully internally and strongly contracted multireference configuration interaction procedures, J. Chem. Phys. 145 (5) (2016) 054104 (2016). doi:10.1063/1.4959029.

[48] F. Neese, Software update: the orca program system, version 4.0, WIREs Comput. Mol. Sci. 8 (1) (2018) e1327 (2018). doi:10.1002/wcms.1327.

[49] F. Sicilia, L. Blancafort, M. J. Bearpark, M. A. Robb, New algorithms for optimizing and linking conical intersection points, J. Chem. Theory Comput. 4 (2) (2008) 257-266 (2008). doi:10.1021/ct7002435. 